\title{
Separating photoluminescence of porous Si in porous colloidal Si and chlorophyll dispersion
}

\author{
Kimihisa Matsumoto $^{1 *}$, Akira Eda ${ }^{1}$, Kazuhide Kamiya ${ }^{1}$, So Ito ${ }^{1}$ \\ 1 Department of Intelligent Robotics, Toyama Prefectural University, Imizu, Toyama 939-0398, Japan \\ *Corresponding author: e-mail: matsu@pu-toyama.ac.jp
}

\begin{abstract}
We observed the luminescence of a chlorophyll and porous Si dispersion using the wavelengthselected excitation and time-gated photoluminescence methods to eliminate the autofluorescence of chlorophyll. Although the photoluminescence (PL) of chlorophyll was dominant when the chlorophyll and porous Si dispersion was excited using the $405 \mathrm{~nm}$ line, the autofluorescence of chlorophyll drastically decreased when the dispersion was excited using the $355 \mathrm{~nm}$ line. During the time-gated photoluminescence experiments, the autofluorescence of chlorophyll disappeared and the broadband PL that corresponded to porous Si was clearly observed. Thus, porous Si could be a very useful luminescent probe for plant imaging when using the wavelength-selected excitation and time-gated photoluminescence methods.
\end{abstract}

Key words: porous Si, photoluminescence, chlorophyll, time-gated PL

\section{INTRODUCTION}

Fluorescence imaging methods using exogenous fluorescent probes are very useful for biomedical and plant pathology research [1]. When plants are analysed using fluorescence imaging, luminescent probes, such as green fluorescent protein and semiconductor quantum dots are employed to visualise targeted diseased tissue. However, plant chloroplasts exhibit very strong autofluorescence at approximately $680 \mathrm{~nm}$ [2, 3]. Autofluorescence of plants is + mainly attributed to the luminescence of light-excited chlorophyll and energy transfer form carotenoids $[4,5]$. To obtain clear signals form exogenous luminescent probes, autofluorescence, as undesired back ground noise, should be eliminated. Therefore, when plant fluorescence imaging experiments are conducted, chlorophyll is generally removed using processes such as etiolation and gene silencing, which allow to obtain clear imaging signals [6]. Since these chlorophyll removal methods damage the plants and result in abnormal physiological conditions, a new florescent imaging method that could be used for live chlorophyll-containing plants is required.

Two optical measurement strategies could be used to eliminate plant autofluorescence: the selective excitation method, which excites only the fluorescence probe (and does not excite chlorophyll) by selecting the wavelength of the excitation source and the time-gated fluorescence imaging method, where the capturing of fluorescence is delayed after excitation $[7,8]$. Fluorescence probes that exhibit broad absorption bands and relatively long luminescent lifetimes are required for these methods.

Porous Si prepared using electrochemical etching presents considerable potential for fluorescence plant imaging owing to its room temperature luminescence, low toxicity, facile preparation, and biodegradability [913]. Furthermore, porous Si presents broad absorption bands owing to its semiconductor band structure and long luminescence lifetime of approximately several microseconds [14]. In one of our previous studies, the surface of porous Si powder was terminated using a carboxylic acid via hydrosilation, which resulted in remarkable improvements in hydrophilicity and luminescence stability [15].

In this study, we investigated the absorption bands and luminescence lifetimes of chlorophyll and carboxylic acid-terminated porous $\mathrm{Si}$ in detail. We also tried to eliminate the luminescence of chlorophyll from the chlorophyll and porous $\mathrm{Si}$ dispersion using selected excitation and time-gated photoluminescence (PL) measurements.

\section{EXPERIMENTAL}

Porous Si powder was prepared by electrochemically etching a Si substrate featuring the resistivity of 1-20 $\Omega$ $\mathrm{cm}$ and the (100) orientation using a mixture of hydrofluoric acid (46-48 \%) and ethanol (99.5\%) (1/1, $\mathrm{v} / \mathrm{v})$. After etching, the porous Si film was milled into fine powder. The as-prepared porous $\mathrm{Si}$ powder was transformed into 10-undecenoic acid-terminated $\mathrm{Si}$ powder via hydrosilation using heat treatment at $80{ }^{\circ} \mathrm{C}$ under Ar atmosphere for $1 \mathrm{~h}$. A plant leaf was cut into pieces and triturated in ethanol to extract chlorophyll. Afterwards, the extracted chlorophyll was mixed with 10undecenoid acid-terminated porous $\mathrm{Si}$.

A Jasco FP 8300 photoluminescence excitation (PLE) spectrometer was used for PLE spectra measurements. A Princeton Instruments PIXIS 256 electrically cooled Si charge-coupled device (CCD) equipped with a Princeton Instruments SP 2150 single monochromator was used for PL spectra measurements, and a NEOARK TC35 $405 \mathrm{~nm}$ $\mathrm{GaN}$ laser was used as excitation source. A Princeton Instruments PI-MAX4 intensified CCD equipped with a single Princeton Instruments SpectraPro SP 2150 monochromator was used for PL decay dynamics and time-gated PL spectra measurements, and a Quantel Ultra 50 third harmonic Nd:YAG laser $(\lambda=355 \mathrm{~nm}, 7 \mathrm{~ns})$ was used as excitation source. 


\section{RESULTS AND DISCCUSION}

Fig. 1 illustrates the PLE spectra of colloidal porous $\mathrm{Si}$ (10-undecenoic acid-decorated porous $\mathrm{Si}$ dispersed in water) and a chlorophyll solution at the detection wavelength of $680 \mathrm{~nm}$. As Si is a semiconductor, porous Si presented a broad absorption band which ranged from 200 to $600 \mathrm{~nm}$ owing to its band structure. On the other hand, the chlorophyll solution could not be excited using wavelengths below $360 \mathrm{~nm}$. As the excitation wavelength increased, the absorption of chlorophyll significantly increased in the 360 to $420 \mathrm{~nm}$ region. Using these PLE spectra, we selected the 355 and $405 \mathrm{~nm}$ lines as excitation lines for the PL spectra and compared the PL and PLE results.

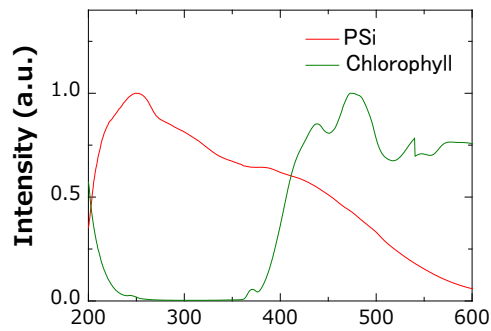

Excitation wavelength (nm)

Fig. 1 PLE spectra of 10-undecenoid acid-terminated porous Si (PSi) and chlorophyll.
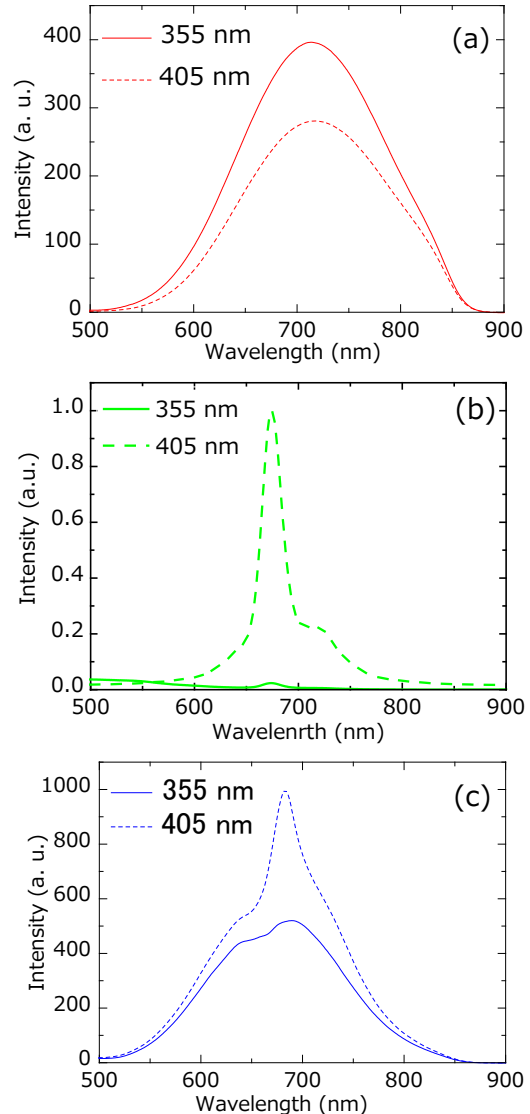

Fig. 2 Room temperature PL spectra at 355 and $405 \mathrm{~nm}$. (a) 10-undecenoid acid-terminated porous $\mathrm{Si}$ (b) chlorophyll and (c) chlorophyll and porous Si dispersion.
Fig. 2(a) presents the room temperature PL spectra of colloidal porous Si excited using the 355 and $405 \mathrm{~nm}$ lines. Broad Gaussian-shaped peaks were observed in the redinfrared region of the spectrum. The different excitation wavelengths did not cause any remarkable changes in the wavelengths and intensities of the PL peaks. Althought The size of the porous Si were not observed at present, there several research about size of luminescent porous $\mathrm{Si}$ by TEM method [16]. Fig. 2(b) depicts the PL spectra of the chlorophyll solution excited using the 355 and $405 \mathrm{~nm}$ lines. When chlorophyll was excited using the $405 \mathrm{~nm}$ line, a sharp, strong PL peak at $680 \mathrm{~nm}$, was observed, and its shape and peak wavelength were attributed to chlorophyll [2-3]. When the chlorophyll solution was excited using the $355 \mathrm{~nm}$ line, the PL intensity drastically decreased and the weak peak at $680 \mathrm{~nm}$ was observed. The PL quenching of chlorophyll was consistent with the PLE results. Chlorophyll could not be efficiently excited using wavelengths below $360 \mathrm{~nm}$. The PL spectra of the chlorophyll and porous Si dispersion are presented in Fig. 2(c). When the dispersion was excited using the $405 \mathrm{~nm}$ line, the PL spectra consisted of superimposed broad Gaussian and sharp peaks at $680 \mathrm{~nm}$, which indicated that both porous $\mathrm{Si}$ and chlorophyll contributed to the luminescence. However, when the dispersion was excited using the $350 \mathrm{~nm}$ line, the PL spectrum mainly consisted of the broad Gaussian peak, which was attributed porous Si. These results indicated that the selected $355 \mathrm{~nm}$ excitation wavelength was useful for reducing the autofluorescence of chlorophyll in the PL spectra.
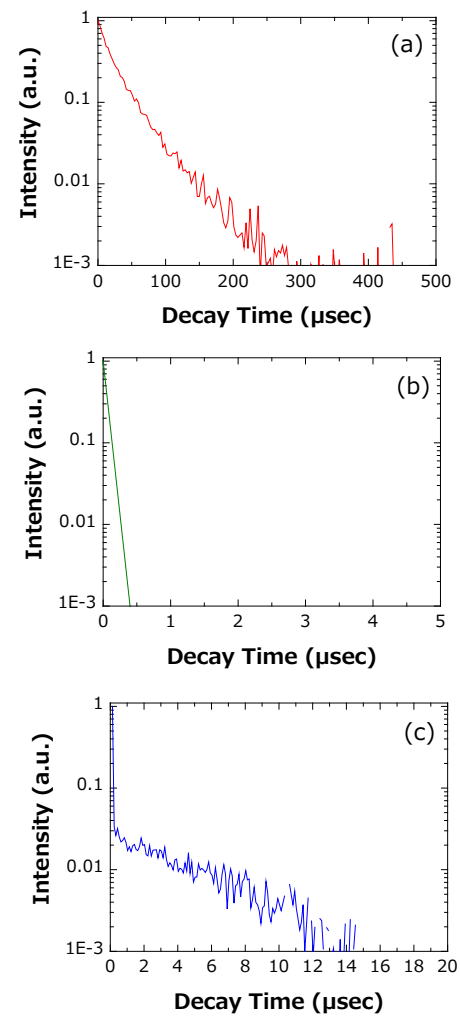

Fig. 3 PL decay dynamics of (a) porous Si powder, (b) chlorophyll and (c) chlorophyll and 10-undecenoic acidterminated colloidal porous Si dispersion 
The PL decay curves of porous $\mathrm{Si}$ at $700 \mathrm{~nm}$ are illustrated in Fig. 3(a). The lifetime of porous $\mathrm{Si}$ was evaluated from the decay curves to be in the tens of microseconds range. This value was similar to that of conventional luminescent Si nanomaterials. Fig. 3(b) presents the PL decay curves of the chlorophyll solution at $700 \mathrm{~nm}$. Because the lifetime of chlorophyll was much shorter: $>0.5 \mu \mathrm{s}$, the detail dynamics of the decay in nanosecond order were not observed using our experimental set up. Fig. 3(c) presents the PL decay curves of the porous Si and chlorophyll dispersion at 700 $\mathrm{nm}$. The curves consisted of two parts: fast decay, which occurred within less than $0.5 \mu \mathrm{s}$, and long decay, which lasted tens of microseconds. The fast and long decays were attributed to the luminescence of chlorophyll and porous $\mathrm{Si}$, respectively. These results indicated that the luminescence of porous Si became dominant $0.5 \mu$ s after the excitation of the porous $\mathrm{Si}$ and chlorophyll dispersion.
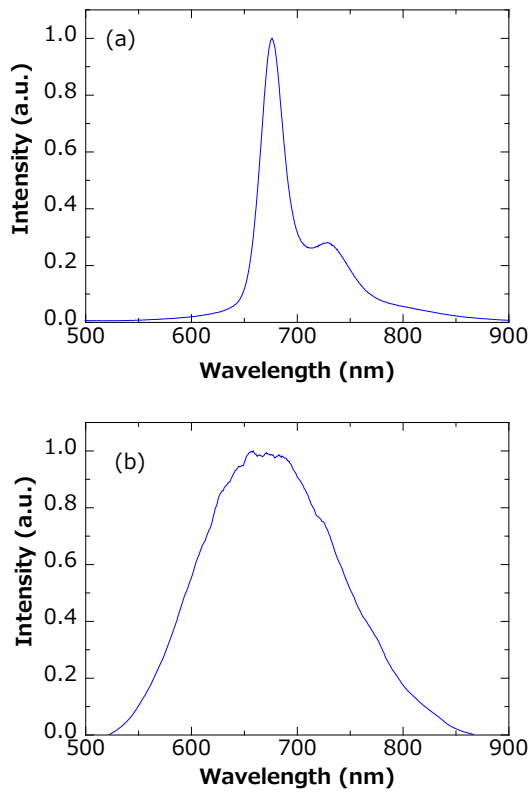

Fig. 4 Time-gated PL spectra of the chlorophyll and colloidal 10-undecenoic acid-terminated porous Si (a) within $0.5 \mu$ s and (b) after $0.5 \mu$ s of excitation.

Fig. 4(a) presents the time-gated PL spectra of the porous $\mathrm{Si}$ and chlorophyll dispersion within $0.5 \mu$ s after excitation using the $355 \mathrm{~nm}$ line of the pulsed laser. The shape of the spectra was similar to that of chlorophyll and no broad PL peaks that could have been attributed to porous Si were observed. The time-gated PL spectra of the porous $\mathrm{Si}$ and chlorophyll dispersion after $0.5 \mu$ s of excitation are presented in Fig. 4(b). The sharp peak at $780 \mathrm{~nm}$, which was attributed to the chlorophyll luminescence clearly disappeared and only the broad Gaussian peak was observed. Only luminescence from porous $\mathrm{Si}$ was observed in the time-gated PL spectra because the spectra was measured after the luminescence of chlorophyll decayed. Additionally, in the case of timegated PL measurements (not PL decay observation), small acquisition time less than $1 \mathrm{~s}$ is advantage for actual bio-imaging. These results indicated that the time-gated luminescence of porous $\mathrm{Si}$ was very useful as fluorescence probe for plant imaging as it allowed to eliminate the autofluorescence of chlorophyll.

4. CONCLUSION

Wavelength-selected excitation and time-gated PL measurements of the porous $\mathrm{Si}$ and chlorophyll dispersion were performed to eliminate the autofluorescence of chlorophyll. The PL of chlorophyll drastically decreased when the dispersion was excited at $355 \mathrm{~nm}$. From the PL decay curves, it was concluded that the PL lifetime of porous $\mathrm{Si}$ was of the order of tens of microseconds, which was much longer than that of chlorophyll. The Gaussianshaped PL peaks of porous Si were observed using the time-gated method by measuring the PL spectra of the porous $\mathrm{Si}$ and chlorophyll dispersion after $0.5 \mu \mathrm{s}$ of excitation. These results indicated that the time-gated measurements of the porous $\mathrm{Si}$ and chlorophyll dispersion could be a potential method for eliminating the autofluorescence of chlorophyll in plants imaging.

References

[1] A. Schulte, I. Lorenzen, M. Böttcher and C. Plieth, Plant Methods, (2006)

[2] O. Björkman and B. Demmig, Planta, 170, 489-504 (1987).

[3] H. K. Lichtenthaler, M. Lang, M. Sowinska, F. Heisel and J. A. Miehé, J. Plant Physiol., 148, 599-612 (1996)

[4] R. Emerson and E. Rabinowitch, Plant Physiol, 35, 477-485 (1960)

[5] K. Inada, Jpn. J. Crop. Sci., 49, 286-294 (1980)

[6] M. Fryer, K. Oxborough, P. Mullineaux and N. Baker, J. Exp. Bot., 53, 1249-1254, (2002)

[7] M. Berezin, W. J. Akers, K. Guo, G. Fischer, E. Daltrozzo, A. Zumbusch and S. Achilefu, Biophys. J., 97, L22-L24 (2009)

[8] L. Gu, D. Hall, Z. Qin, E. Anglin, J. Joo, D. Mooney and M. Sailor, Nat Commun. J., 4, 2326 (2013)

[9] Y. Kodama, PLoS One, 0152484 (2016)

[10] L. T. Canham, Appl. Phys. Lett., 57, 1046-1048 (1990)

[11] K. Cheng and R. Anthony, Nano Lett., 11, 1952$1956(2011)$

[12] D. Kovalev, E. Gross, N. Künzner, F. Koch, V. Yu. Timoshenko and M. Fujii, Phys. Rev. Lett., 89, 137401 (2002)

[13] Z. F. Li and E. Ruckenstein, Nano. Lett., 4,14631467 (2004)

[14] C. Garcia, B. Garrido, P. Pellegrino, R. Ferre, J. A. Moreno, and J. R. Morante, Appl. Phys. Lett., 82, 1595 (2003)

[15] M. Sakakibara, K. Matsumoto, K. Kamiya, S. Kawabata, M. Inada and S. Suzuki, Jpn. J. Appl. Phys., 57, 02CB16 (2018)

[16] A. G. Cullis and L. T. Canham, Nature 353, 335 (1991)

(Received April 6, 2019; Accepted July 24, 2019; Published Online October 1, 2019) 